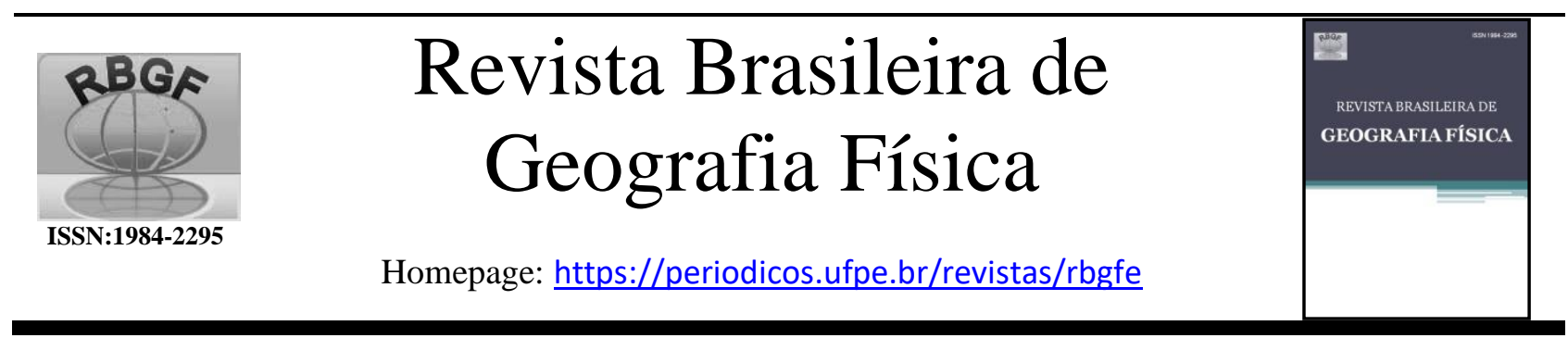

\title{
Tendências em índices extremos de precipitação em Cabaceiras (PB) para períodos distintos
}

\author{
Rafaela Lisboa Costa ${ }^{1}$, Heliofábio Barros Gomes ${ }^{2}$, Fabrício Daniel dos Santos Silva ${ }^{3}$, Rodrigo Lins da Rocha Júnior ${ }^{4}$, \\ Giuliene Carla dos Santos Silva ${ }^{5}$, William Max de Oliveira Romão ${ }^{6}$
}

\begin{abstract}
${ }^{1}$ Dra. em Geociências Aplicadas, Bolsista PNPD/CAPES, Instituto de Ciências Atmosféricas, Universidade Federal de Alagoas, Av. Lourival Melo Mota, s/n, Tabuleiro do Martins, CEP:57072-900, Maceió-AL. (82) 3214-1367. rafaelalisboac@ gmail.com. ${ }^{2}$ Professor Associado, Instituto de Ciências Atmosféricas, Universidade Federal de Alagoas, Av. Lourival Melo Mota, s/n, Tabuleiro do Martins, CEP:57072-900, Maceió-AL. (82) $3214-1367$. heliofabio@icat.ufal.br. ${ }^{3}$ Professor Adjunto, Instituto de Ciências Atmosféricas, Universidade Federal de Alagoas, Av. Lourival Melo Mota, s/n, Tabuleiro do Martins, CEP:57072-900, Maceió-AL. (82) 3214-1367. fabricio.santos @ icat.ufal.br. (autor correspondente) ${ }^{4}$ Mestrando em Meteorologia, Instituto de Ciências Atmosféricas, Universidade Federal de Alagoas, Av. Lourival Melo Mota, s/n, Tabuleiro do Martins, CEP:57072-900, MaceióAL. (82) 3214-1367. rrodrigo.junior@ hotmail.com. ${ }^{5}$ Graduanda em Meteorologia, Instituto de Ciências Atmosféricas, Universidade Federal de Alagoas, Av. Lourival Melo Mota, s/n, Tabuleiro do Martins, CEP:57072-900, Maceió-AL. (82) 3214-1367. giucarla.98@ gmail.com. ${ }^{6}$ Graduando em Meteorologia, Instituto de Ciências Atmosféricas, Universidade Federal de Alagoas, Av. Lourival Melo Mota, s/n, Tabuleiro do Martins, CEP:57072900, Maceió-AL. (82) 3214-1367. william.romao@icat.ufal.br.
\end{abstract}

Artigo recebido em 01/08/2019 e aceito em 26/01/2020

\section{R E S U M O}

Este trabalho teve como objetivo aplicar e analisar 11 índices de extremos de precipitação formulados pelo ETCCDI (Expert Team on Climate Change Detection and Indices, www.clivar.org/organization/etccdi), para a cidade de Cabaceiras, localizada na mesorregião da Borborema e microrregião do Cariri Oriental paraibano. Município integrante da região semiárida, tem o título de município onde menos chove no Brasil, com média anual de pouco mais de 300mm. Foram utilizados dados diários de precipitação contínuos de 90 anos para os índices de extremos, com a série temporal analisada para quatro períodos distintos, o comprimento total da série, 1928 a 2017, assim como três segmentos de 30 anos (1928-1957, 1958-1987 e 1988-2017). Os resultados evidenciaram que para muitos índices, tendências opostas e significativas podem ser observadas a depender do subperíodo estudado, assim como haver diferença entre estas tendências e as obtidas ao analisar-se o período total dos dados. Os índices R1, R10 e R20mm mostram tendências significativas negativas no subperíodo 1928-1957, porém positivas nos dois subperíodos seguintes, refletindo em uma tendência positiva significativa no período total de 1928 a 2017. Outros exemplos interessantes são relativos aos índices CDD para dias secos consecutivos e PRCPTOT, para a precipitação total anual com chuvas superiores a $1 \mathrm{~mm}$. O CDD apresentou tendência positiva significativa apenas no subperíodo 1928-1957, porém negativas não significativas nos subperíodos posteriores, refletindo em tendências negativa não significativa no comprimento total da série. $\mathrm{O}$ índice PRCPTOT mostra comportamento oposto ao índice CDD, com tendência negativa significativa no subperíodo 19281957, positiva em 1958-1987 e novamente negativa em 1988-2017, porém para o comprimento total da série a tendência e positiva e significativa. Tais resultados evidenciam que a análise de tendências de extremos é perceptivelmente sensível a amostra do período analisado, podendo não refletir a realidade da série temporal quanto maior for o comprimento total da série temporal, e precisam ser utilizados com cautela.

Palavras-chave: variabilidade climática, períodos secos e úmidos, semiárido.

\section{Trends in extreme precipitation indexes in Cabaceiras (PB) for different periods}

\section{A B S T R A C T}

This work aimed to apply and analyze 11 precipitation extremes indexes formulated by ETCCDI (Expert Team on Climate Change Detection and Indices, www.clivar.org/organization/etccdi), for the city of Cabaceiras, located in the Borborema mesoregion and microregion of Paraíba Oriental Cariri. A municipality in the semiarid region, it has the title of municipality where it rains less in Brazil, with an annual average of just over $300 \mathrm{~mm}$. Daily 90 -year continuous precipitation data were used for the extreme indices, with the time series analyzed for four distinct periods, the total length of the series, 1928 to 2017, as well as three 30-year segments (1928-1957, 1958- 1987 and 1988-2017). The results showed that for many indices, opposite and significant trends can be observed depending on the sub period studied, as well as differences between these trends and those obtained by analyzing the total data period. The R1, R10 and R20mm indices show significant negative trends in the 1928-1957 sub period, but positive in the following two sub periods, 
reflecting a significant positive trend in the total period from 1928 to 2017. Other interesting examples are CDD indices for consecutive dry days, and PRCPTOT, for total annual rainfall with rainfall greater than $1 \mathrm{~mm}$. The CDD showed significant positive trend only in the 1928-1957 sub period, but non-significant negative trends in the subsequent sub periods, reflecting non-significant negative trends in the total length of the series. The PRCPTOT index shows behavior opposite to the CDD index, with a significant negative trend in the 1928-1957 sub period, positive in 1958-1987 and negative again in 1988-2017, but for the total length of the series the trend is positive and significant. These results show that the analysis of extreme trends is noticeably sensitive to the sample of the analyzed period, and may not reflect the reality of the time series the longer the total length of the time series, and need to be used with caution.

Keywords: climate variability, dry and wet periods, semiarid.

\section{Introdução}

Eventos climáticos extremos podem ocorrer de diferentes formas, por exemplo, como inundações, secas prolongadas e ondas de calor. A humanidade tem desenvolvido ao longo do tempo uma boa compreensão da frequência de eventos climáticos extremos e as localizações geográficas onde eles são mais prováveis de acontecer. Precisase entender as diferenças entre os eventos extremos de tempo e de clima. Na região Nordeste do Brasil, o fenômeno climático que causa os maiores prejuízos à população, especialmente a do semiárido, é a seca, que varia em intensidade, magnitude e frequência (Cunha et al., 2015; Cunha et al., 2018; Marengo et al., 2018; Alvalá et al., 2017; Martins et al., 2018; Martins et al., 2018). Eventos extremos de tempo estão relacionados à mudanças repentinas das condições meteorológicas em um dia, como enchentes causadas por fortes temporais (Alves et al., 2017), ou a um curto período de tempo, como ondas de calor ou frio (Geirinhas et al., 2017).

Estudar a tendência de índices de extremos climáticos a partir de dados diários de precipitação é uma forma de avaliar se determinado local/região está sendo exposto a possíveis mudanças espaçotemporais no comportamento desta variável (Nóbrega et al., 2015). Além disso, os potenciais impactos das mudanças climáticas na vida humana, economia social e ecossistemas naturais são sentidos mais fortemente através das variações de extremos (Jiang et al., 2011).

O crescente interesse em estudar índices de extremos climáticos é mundial. Para citar algumas pesquisas, Croitoru et al. (2016) avaliaram as mudanças nos índices de extremos de precipitação na Romênia no período de 1961-2013, percebendo o incremento significativo da contribuição de eventos diários extremos para a precipitação total anual. Keggenhoff et al. (2014) analisaram os mesmos índices para a Geórgia, e notaram também o aumento no número de dias úmidos $\mathrm{e}$ extremamente úmidos, no entanto, a precipitação total anual diminuiu, evidenciando a concentração de dias mais chuvosos em detrimento do total anual precipitado. Ambientes remotos, como a Península Antártica, tiveram seus registros de precipitação a partir de uma estação de observação estudados entre 1969 a 2010, evidenciando diminuição das chuvas neste período em quase todos os meses do ano (Wanderley et al., 2016).

O IPCC (2013) em seu quinto relatório de avaliação mostra que muitos estudos regionais têm analisado as mudanças nos extremos climáticos e têm, geralmente, identificado aumento na intensidade de chuvas que ultrapassam acumulados horários/diários considerados limite para o sistema de drenagem urbana das cidades.

$\mathrm{O}$ NRB tem sido bem explorado em estudos envolvendo análises de índices de mudanças climáticas, no entanto, de maneira fragmentada. Santos e Brito (2007) estudaram apenas os índices relacionados à precipitação em dois estados da região Nordeste, Rio Grande do Norte e Paraíba, para o período 1950 a 2000. Santos et al. (2009) estudaram tendências dos índices de precipitação, mas apenas para o estado do Ceará. Silva e Azevedo (2008) e Silva et al. (2012) estudaram as tendências dos índices de mudanças climáticas para o estado da Bahia. Nóbrega et al. (2015), estudaram a variabilidade e tendências de índices de extremos de chuva em Pernambuco por meio de dados gradeados do Climate Prediction Center (CPC) relativos ao período de 1978 a 2010. Dantas et al. (2015) estudaram tendências sazonais e anuais de índices de extremos para a Cidade de Campina Grande, no período 1975 a 2011.

Regiões semiáridas, como no interior do Nordeste do Brasil, estão entre as mais vulneráveis a adversidades climáticas e extremos (Guimarães et al., 2016). Percebe-se dos artigos citados no parágrafo anterior, que o que mais difere entre todos os estudos é o período de análise dos dados, a maior parte deles a partir das décadas de $1950 \mathrm{e}$ 1960 , e poucos deles conseguiram contemplar os anos recentes de seca no Nordeste, de 2012 a 2017. Um que contempla dados de maior comprimento é o estudo de Nóbrega et al. (2014), que estudos eventos extremos de precipitação na Paraíba entre 1935 e 2004, porém para todo o comprimento da série. Neste contexto, o objetivo deste trabalho é avaliar a tendência de índices extremos de precipitação em Cabaceiras, no Estado da Paraíba, 
considerada uma das cidades brasileiras com menor pluviometria média anual, por meio da análise do comportamento das tendências de 11 índices de extremos de precipitação ao longo de 90 anos de dados diários de chuvas, entre 1928 e 2017, além de estudar dentro destes 90 anos, três segmentos distintos de 30 anos (1928-1957; 19581987; 1988-2017), a fim de identificar períodos se há diferentes comportamentos nas tendências a depender do período específico de análise.

\section{Material e métodos}

Neste estudo foram trabalhados dados diários de precipitação de 90 anos (1928-2017) da cidade de Cabaceiras (PB), para estudar alterações climáticas recentes por meio da aplicação de 11 índices de extremos de precipitação recomendados pela Comissão de Climatologia da Organização Meteorológica Mundial (WMO-CCL de suas siglas em inglês) - e pelo projeto de pesquisa sobre Variabilidade Climática do Programa Mundial de Pesquisas do Clima (CLIVAR-WCRP de suas siglas em inglês) (Tank et al., 2009; Zwiers et al., 2011; Zhang et al., 2011).

Os dados são oriundos do Departamento Nacional de Obras Contra as Secas (DNOCS), que instalou e coletou os dados pluviométricos desde o início da série, e da Agência Executiva de Águas do Estado da Paraíba (AESA), que administra atualmente a coleta de dados pluviométricos no Estado da Paraíba.

A localização geográfica de Cabaceiras é mostrada na Figura 1, na Região Geográfica Imediata de Campina Grande, a aproximadamente 300 metros de altitude. Segundo Alves et al. (2017), seu clima é classificado como DA'd, semiárido megatérmico com excedente hídrico pequeno ou nulo, de acordo com a classificação climática de Thornthwaite e Mather (1955). Já segundo Medeiros et al. (2012), o clima da área de estudo é considerado do tipo As - clima quente, de acordo com a classificação de Koppen.

Os índices de extremos foram calculados usando o software RClimDex (Zhang et al., 2004). Nos índices, foram observados parâmetros estatísticos como a tendência linear calculada pelo método dos mínimos quadrados e o nível de significância estatística das tendências, estimado pelo teste t-student (valor-p) (Baez-Gonzaleza et al., 2018). Esses resultados foram discutidos para três subperíodos de 30 anos entre 1928 e 2017, e para o período total de 90 anos dos dados. A tabela 1 mostra a descrição dos índices climáticos.

Os índices são obtidos analisando extremos para cada ano independente da série temporal de dados, formando-se uma nova série temporal para cada índice por ano. RX1Day e RX5Day são relativos aos extremos diários e acumulado em 5 dias consecutivos de chuva por ano. Os índices R95p e R99p são relativos ao acumulados de chuvas anuais que ultrapassem os percentis 95 e 99 da distribuição dos dados, por exemplo, se o valor do percentil 95 para as chuvas diárias de uma localidade é $50 \mathrm{~mm}$, se houver no ano três eventos de chuvas diárias superiores a este valor, elas serão somadas e o valor representativo do índice R95p para este determinado ano será este, e o mesmo acontece para o índice R99p. O PRCPTOT, assim como para todos os outros índices, só considera as chuvas maiores ou iguais ao valor de $1 \mathrm{~mm}$, e representa esta soma de todos os eventos anuais a partir deste valor de referência. O SDII é um índice de proporcionalidade, onde soma-se o total anual de chuvas a partir de $1 \mathrm{~mm}$, e divide-se este valor pelo número de dias com chuvas para cada ano, dando uma medida de quão boa ou má pode ser a distribuição temporal da chuva anual. Os índices R1mm, R10mm e R20mm são contagens simples de quantos eventos por ano ocorrem a partir deste limiares. Os índices CDD e CWD são opostos, o CDD indica, em número total de dias seguidos por ano, a maior sequência de não ocorrência de chuvas entre dois eventos de pelo menos $1 \mathrm{~mm}$, e o índice CWD fornece o número máximo consecutivos de dias com precipitação a cada ano, sempre levando em consideração o valor de referência de ao menos $1 \mathrm{~mm}$ por dia. Valores inferiores a $1 \mathrm{~mm}$, ou seja, de $0,1 \mathrm{~mm}$ a $0,9 \mathrm{~mm}$, não são considerados no cômputo dos índices e são desprezados da série temporal.

A Tabela 1 mostra de forma resumida a sigla de cada índice, o que cada índice representa em termos do que calcula, e a unidade, que pode ser em $\mathrm{mm}, \mathrm{mm} / \mathrm{dia}$, e dias. 
Tabela1. Índices de extremos climáticos derivados da precipitação diária, com suas definições e unidades. PRCP é o valor da precipitação diária. $\mathrm{PRCP} \geq 1 \mathrm{~mm}$ representa um dia úmido e $\mathrm{PRCP}<1 \mathrm{~mm}$, um dia seco.

\begin{tabular}{ccc}
\hline \multicolumn{1}{c}{ Índice } & Definição do Índice & Unidade \\
\hline RX1DAY & Precipitação máxima em um dia & $\mathrm{mm}$ \\
RX5DAYS & Maior acumulação de precipitação em cinco dias consecutivos no ano & $\mathrm{mm}$ \\
R95P & Precipitação total anual quando PRCP> percentil 95 & $\mathrm{~mm}$ \\
R99P & Precipitação total anual quando PRCP> percentil 99 & $\mathrm{~mm}$ \\
PRCPTOT & Precipitação total anual nos dias com PRCP $\geq 1 \mathrm{~mm}$ & $\mathrm{~mm}$ \\
SDII & Precipitação total anual dividida pelo número de dias com PRCP $\geq 1 \mathrm{~mm}$ & $\mathrm{~mm} /$ dia \\
R1mm & Contagem anual de dias quando PRCP $\geq 1 \mathrm{~mm}$ & Dias \\
R10mm & Contagem anual de dias quando PRCP $\geq 10 \mathrm{~mm}$ & Dias \\
R20mm & Contagem anual de dias quando PRCP $\geq 20 \mathrm{~mm}$ & Dias \\
CDD & Comprimento máximo da estiagem, ou número máximo de dias consecutivos com & Dias \\
CWD & PRCP<1 mm & Dias \\
& Comprimento máximo de período úmido, ou número máximo de dias consecutivos & com PRCP> 1 mm \\
\hline
\end{tabular}

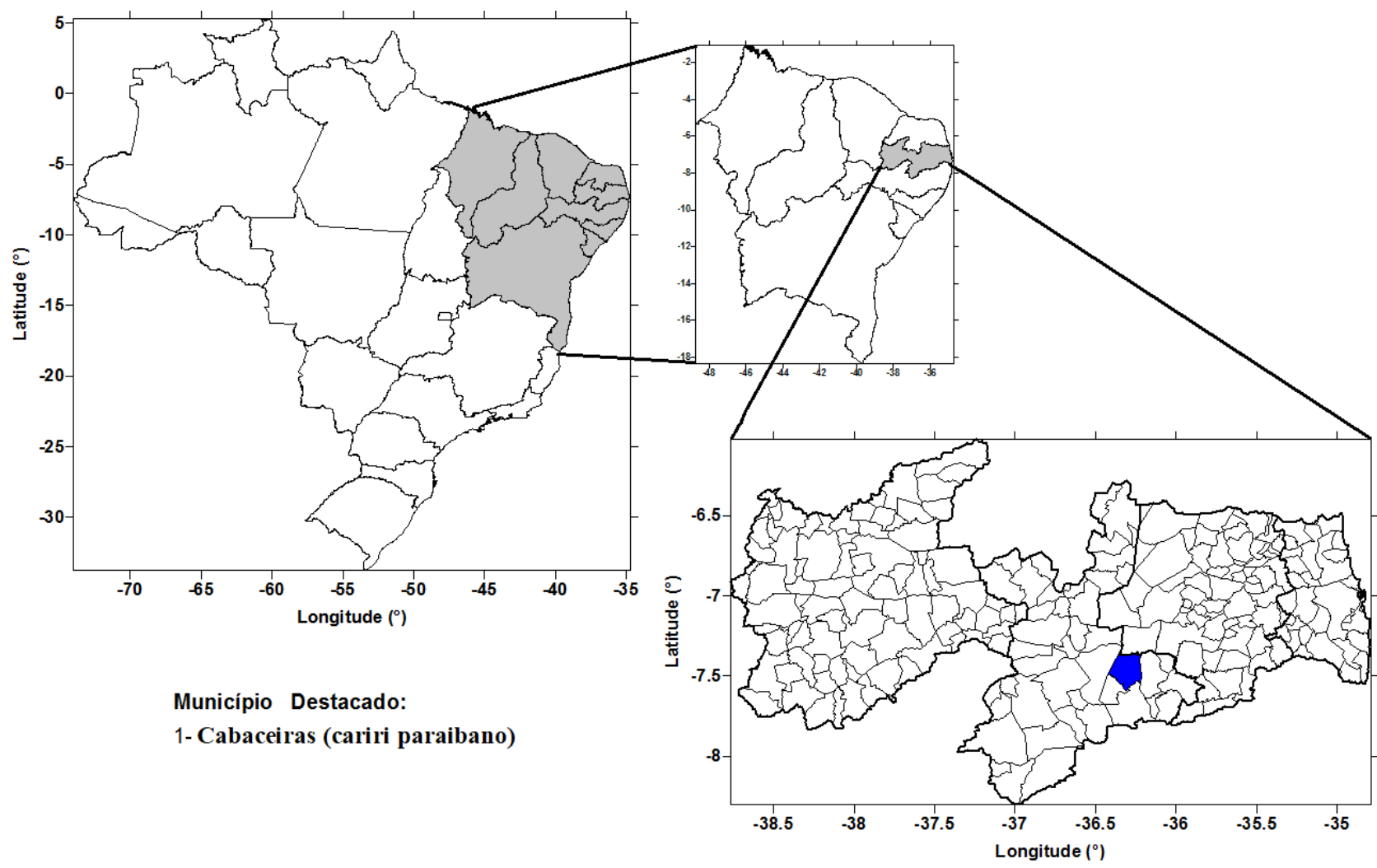

Figura 1. Localização geográfica do município de Cabaceiras, a $-7,49^{\circ}$ de latitude e $-36,29^{\circ}$ de longitude.

\section{Resultados e discussão}

Um obstáculo para a análise de dados de longa data são as falhas nas observações. A série temporal total de precipitação Cabaceiras de 1928 a 2017 compreende 32868 registros diários, dos quais apenas 2,04\% apresentaram ausência de informações, o que de forma alguma comprometeria as análises desta pesquisa. Mesmo assim, as falhas foram preenchidas de acordo com a metodologia proposta e descrita por Carvalho et al (2016).

Costa, R, L.; Gomes, H. B.; Silva, F. D. S.; da Rocha Júnior, R. L.; Silva, G. C. S.; Romão, W. M.
A Tabela 2 mostra que, dos 11 índices estudados, apenas 4 apresentaram tendências significativas e positivas na extensão total da série entre 1928 a 2017: R1mm, R10mm, R20mm e PRCPTOT. Outros 3 índices apresentaram tendências estatisticamente significantes em períodos específicos: RX5DAY, CDD e CWD.

No subperíodo 1928-1957, os índices RX5DAY, R1mm, R10mm, R20mm, CWD e PRCPTOT apresentaram tendências significativas e negativas, enquanto o índice CDD apresentou forte tendência positiva com significância 
estatística. Isto indica que os primeiros 30 anos da série de dados foi marcada pela redução das chuvas entre o início e o fim da série. Esta situação se inverte no subperíodo 1958-1987, apesar de apenas 2 índices apresentarem tendências positivas significativas, $\mathrm{R} 1 \mathrm{~mm}$ e CWD, mostrando $\mathrm{o}$ incremento no número de dias com chuva superior a $1 \mathrm{~mm}$ e o aumento do número de dias úmidos consecutivos. Em 1988-2017, nenhum dos índices apresentou tendência com significância estatística, predominando tendências negativas como em 1928-1957.

Serão mostradas e discutidas apenas as figuras dos resultados relativos aos índices que apresentaram, para ao menos um dos períodos analisados, tendências com significância estatística ao nível de confiança de $95 \%$ pelo teste t-student (P_Valor $<0,05)$.

A Figura 2 mostra os gráficos obtidos para o índice RX5DAY. Este índice apresentou tendência estatisticamente significante apenas para os primeiros 30 anos da série, 1928-1957, com redução de 2,6mm/ano para acumulados de precipitação a partir de 5 dias consecutivos (Figura 2a). Em contrapartida, para os dois subperíodos seguintes e para o período total da série, as tendências não apresentaram significância estatística, com os respectivos valores: $0,6 \mathrm{~mm} / \mathrm{ano}$ em 1958-1987, -0,07mm/ano em 1988-2017, e 0,23mm/ano para 1928-2017.

As Figuras 3 e 4 mostra os gráficos obtidos para os índices R10mm e R20mm, que representam a contagem de eventos acima destes limiares. Este índice apresentaram comportamento similar com tendências de redução nos períodos 1928-1957 (Figura 3a e Figura 4a), de aproximadamente -9,6 e -3,6 dias no período, respectivamente, porém positivas no período completo da série (Figura $3 \mathrm{~d}$ e Figura 4d), de aproximadamente 6,9 e 3,2 dias no período, respectivamente. $\mathrm{O}$ índice que apresentou tendências estatisticamente significantes na maior parte dos períodos analisados, negativa de $-37,5$ dias no período 1928-1957 (Figura 5a), e positiva de 23,7 dias para 1958-1987 (Figura 5b), assim como para o período total da série 1928-2017 (Figura 5d) de 13,5 dias, foi a contagem anual de dias com precipitações superiores a $1 \mathrm{~mm}(\mathrm{R} 1 \mathrm{~mm})$.

O aumento de dias secos consecutivos CDD, foi estatisticamente significativo apenas para os primeiros 30 anos de análise: 1928-1957 (Figura 6a), de aproximadamente 112 dias no período. Este resultado está associado a grande seca observada entre o final da década de 1940 e a década de 1950, associado de forma evidente a redução do número de dias úmidos consecutivos - CWD (Figura 7a), de aproximadamente 5 dias no período. O sinal da tendência de CWD, no entanto, é o oposto para o
CDD no período 1958-1987 (Figura 7b), 2,2 dias no período versus $-85,4$ dias no mesmo período para o índice CDD.

Por fim, ao analisarmos um dos mais importantes índices, o PRCPTOT, que indica se determinado período teve tendência de aumento ou diminuição das chuvas, encontramos os resultados mais contrastantes. Devido a já comentada, e verificada, grande seca ocorrida entre o da década de 1940 e década de 1950, o primeiro subperíodo analisado, 1928-1957, apresentou uma tendência negativa estatisticamente significativa de $12,7 \mathrm{~mm} /$ ano (Figura $8 \mathrm{a}$ ), ou $-378 \mathrm{~mm}$ no período. Os dois subperíodos seguintes, 1958-1987 (Figura 8b) e 1988-2017 (Figura 8c), alternaram incremento e diminuição da precipitação total anual da ordem de 6,4 e $-3,1 \mathrm{~mm} / \mathrm{ano}$, ou $192 \mathrm{~mm}$ e $92 \mathrm{~mm}$, respectivamente, embora tais tendências não apresentarem significância estatística. Por fim, se apenas o período total da série fosse analisado, 1928-2017 (Figura 8d), tem-se o resultado de tendência positiva, e estatisticamente significante, de $2 \mathrm{~mm} / \mathrm{ano}$, ou $176 \mathrm{~mm}$ no período.

Estes resultados demonstram a importância de analisar com clareza tendências em índices de extremos climáticos, principalmente para a precipitação, que apresenta distribuição no tempo e no espaço bem menos homogênea que a temperatura. Estudos desta natureza precisam deixar claro que suas conclusões podem se referir apenas ao período de tempo em estudo, não podendo-se de forma algumas inferir ou extrapolar as tendências observadas para períodos retrógrados ou futuros.

A região Nordeste é rica em estudos sobre o comportamento de índices de extremos, o que, por sua vez, não esgota as possibilidades de mais pesquisas nesta área. Isto se dá devido aos diferentes tipos de dados utilizados, a qualidade dos dados utilizados, e as diferentes escalas temporais dos estudos. Nóbrega et al. (2015) estudaram estes índices de extremos de precipitação no Estado de Pernambuco para o período 1978-2010, usando séries temporais de precipitação diária advindas da análise gradeada do Climate Prediction Center (CPC); em estudo específico para Campina Grande (PB). Dantas et al. (2015) analisaram os índices a partir de séries temporais do INMET no período 1975-2011. Tais índices têm sido estudados para outras regiões do Brasil, a exemplo de Natividade et al. (2017), que os analisaram para dados observados e sob cenários de mudanças climáticas em Minas Gerais, e Santos e Oliveira, (2017) para o Estado do Pará com dados entre 1970 e 2006.

Para confrontar diretamente resultados obtidos por outros autores com os desta pesquisa, 
pode-se citar o estudo de Santos e Brito. (2007), que utilizou dados pluviométricos de municípios do Rio Grande do Norte e da Paraíba, incluindo-se Cabaceiras, de 1935 a 2000. Para o período total dos dados, encontraram tendência negativa de 0,273 dias/ano para o índice CDD, pouco inferior aos $-0,459$ dias/ano desta pesquisa. Para o índice CWD, encontraram a tendência positiva de 0,004 dias/ano similar aos 0,002 dias/ano desta pesquisa. A tendência positiva do índice SDII, de $0,08 \mathrm{~mm} / \mathrm{dia} /$ ano foi superior aos $0,02 \mathrm{~mm} / \mathrm{dia} / \mathrm{ano}$ desta pesquisa. O índice PRCPTOT apresentou tendência de $4,041 \mathrm{~mm} /$ ano $(266,7 \mathrm{~mm}$ no período), superior a $1,9 \mathrm{~mm} / \mathrm{ano}(175,9 \mathrm{~mm}$ no período $)$ desta pesquisa. Os índices R95p e R99p apresentaram valores de 0,172 e $-0,54 \mathrm{~mm} / \mathrm{ano}$, e nesta pesquisa 0,64 e $0,07 \mathrm{~mm} / \mathrm{ano}$, respectivamente. Os índices RX1Day e RX5Day apresentaram valores de 0,006 e $0,218 \mathrm{~mm} / \mathrm{ano}$, e nesta pesquisa 0,22 e Tabela 2. Inclinação do coeficiente angular da reta (Slope) e respectivo p-valor do teste de hipótese estatístico t-student para indicação da significancia estatística da tendencia linear. Destacados em negrito as tendencias estatisticamente significantes.

\begin{tabular}{ccccccccc}
\hline & \multicolumn{2}{c}{ Período:1928-2017 } & \multicolumn{2}{c}{ Período:1928-1957 } & \multicolumn{2}{c}{ Período:1958-1987 } & \multicolumn{2}{c}{ Período:1988-2017 } \\
\cline { 2 - 9 } Indices & Slope & P_Valor & Slope & P_Valor & Slope & P_Valor & Slope & P_Valor \\
\hline RX1DAY & 0,22 & 0,12 & $-1,24$ & 0,11 & 0,26 & 0,62 & $-0,26$ & 0,77 \\
RX5DAY & 0,27 & 0,17 & $-\mathbf{2 , 5 5}$ & $\mathbf{0 , 0 1}$ & 0,65 & 0,43 & $-0,07$ & 0,96 \\
SDII & 0,02 & 0,27 & $-0,09$ & 0,27 & $-0,04$ & 0,67 & $-0,02$ & 0,76 \\
R10mm & $\mathbf{0 , 0 9}$ & $\mathbf{0 , 0 0}$ & $-\mathbf{0 , 3 2}$ & $\mathbf{0 , 0 0}$ & 0,19 & 0,24 & $-0,13$ & 0,32 \\
R20mm & $\mathbf{0 , 0 4}$ & $\mathbf{0 , 0 0}$ & $\mathbf{- 0 , 1 3}$ & $\mathbf{0 , 0 1}$ & 0,03 & 0,78 & 0,00 & 1,00 \\
R1mm & $\mathbf{0 , 1 4}$ & $\mathbf{0 , 0 1}$ & $\mathbf{- 1 , 2 5}$ & $\mathbf{0 , 0 0}$ & $\mathbf{0 , 8 0}$ & $\mathbf{0 , 0 0}$ & $-0,24$ & 0,34 \\
CDD & $-0,40$ & 0,14 & $\mathbf{3 , 7 2}$ & $\mathbf{0 , 0 2}$ & $-2,85$ & 0,06 & $-0,27$ & 0,81 \\
CWD & 0,00 & 0,69 & $-\mathbf{0 , 1 5}$ & $\mathbf{0 , 0 0}$ & $\mathbf{0 , 0 7}$ & $\mathbf{0 , 0 2}$ & 0,00 & 0,94 \\
R95P & 0,64 & 0,09 & $-3,75$ & 0,06 & 0,88 & 0,64 & 0,16 & 0,95 \\
R99P & 0,07 & 0,76 & $-0,28$ & 0,81 & 0,23 & 0,78 & $-0,73$ & 0,57 \\
PRCPTOT & $\mathbf{1 , 9 9}$ & $\mathbf{0 , 0 1}$ & $\mathbf{- 1 2 , 6 2}$ & $\mathbf{0 , 0 0}$ & 6,39 & 0,11 & $-3,06$ & 0,43 \\
\hline
\end{tabular}



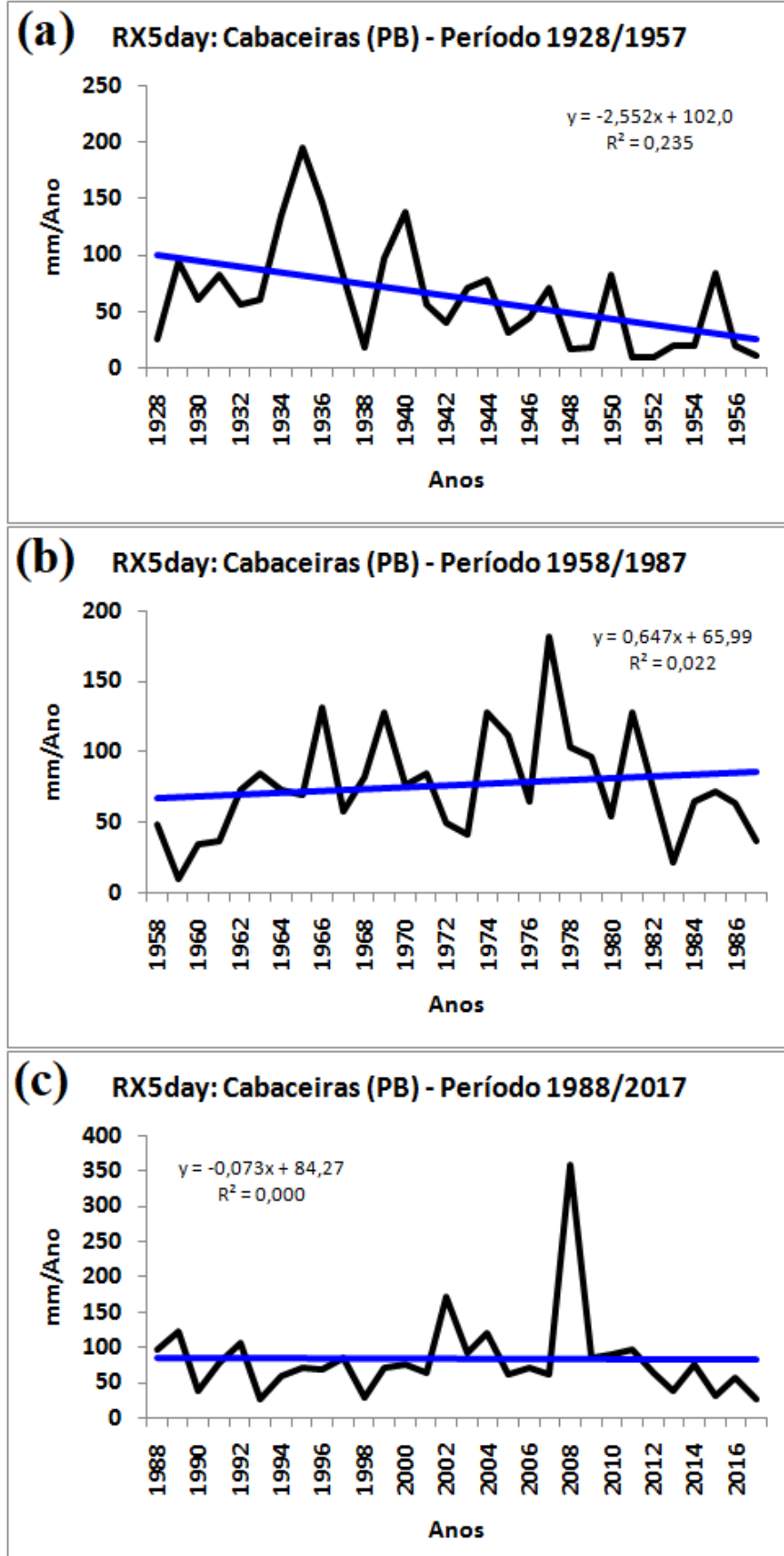

(d) RX5day: Cabaceiras (PB) - Período 1928/2017

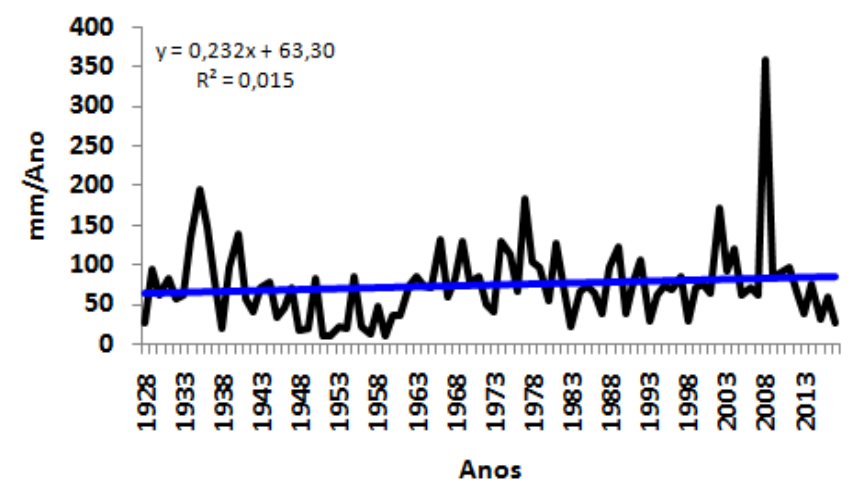

Figura 2. Índice RX5DAY para os subperíodos (a)1928-1957, (b)1958-1987 e (c)1988-2017, e para o período total (d)1928-2017. 
(a) R10mm: Cabaceiras (PB) - Período 1928/1957

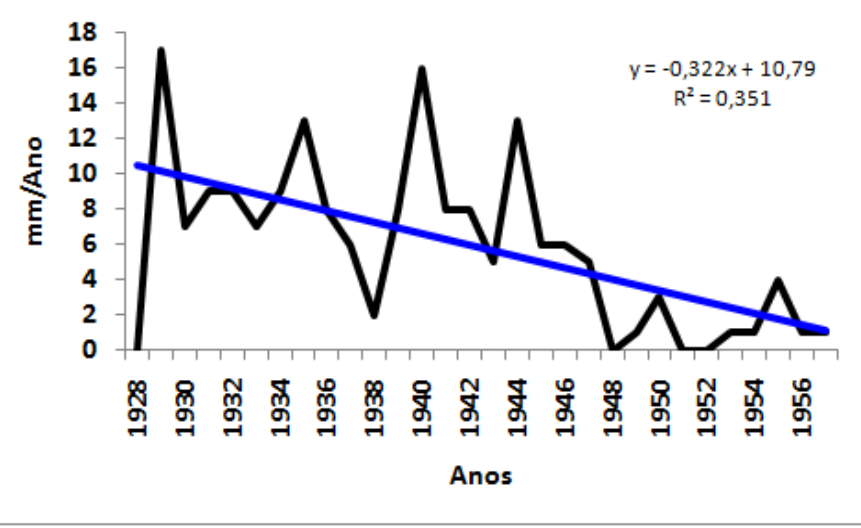

(b) R10mm: Cabaceiras (PB) - Período 1958/1987

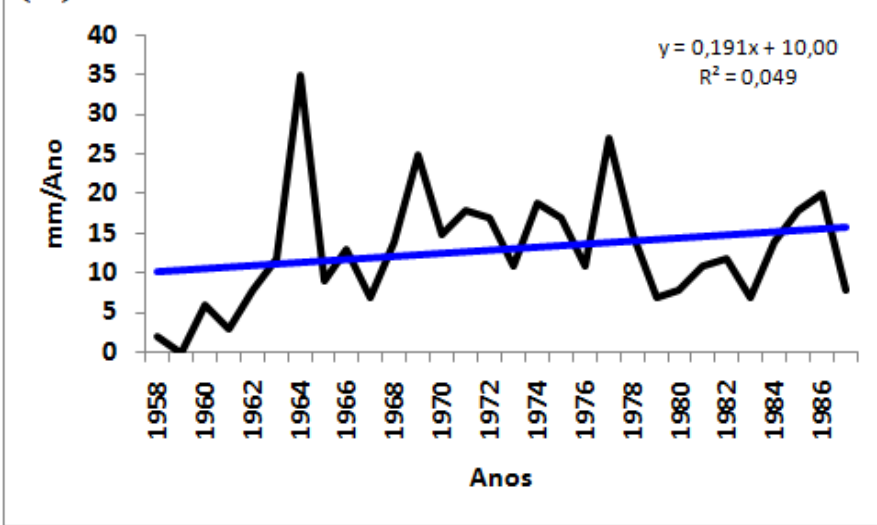

(c) R10mm: Cabaceiras (PB) - Período 1988/2017

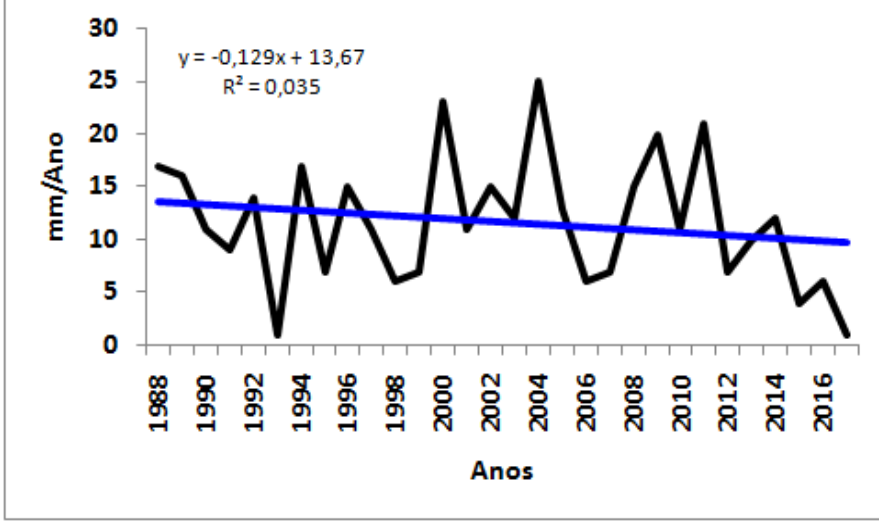

(d) R10mm: Cabaceiras (PB) - Período 1928/2017

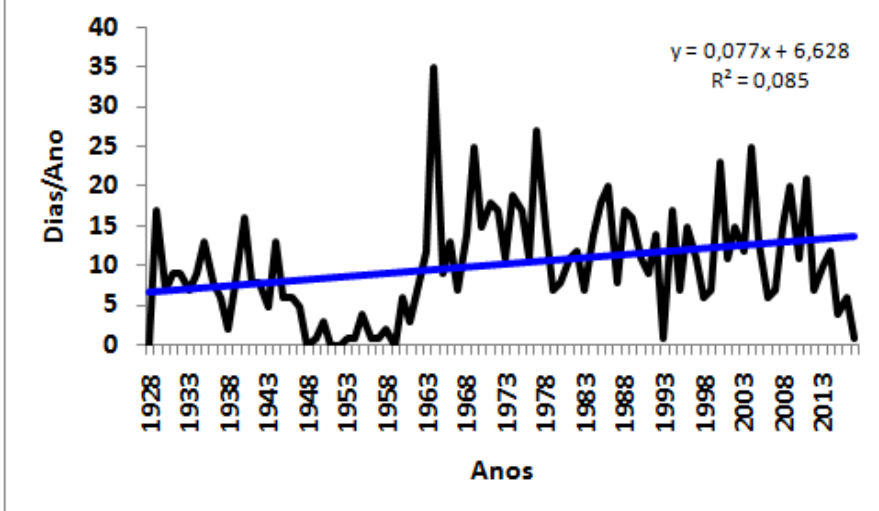

Figura 3. Índice R10mm para os subperíodos (a)1928-1957, (b)1958-1987 e (c)1988-2017, e para o período total (d)1928-2017. 
(a) R20mm: Cabaceiras (PB) - Período 1928/1957

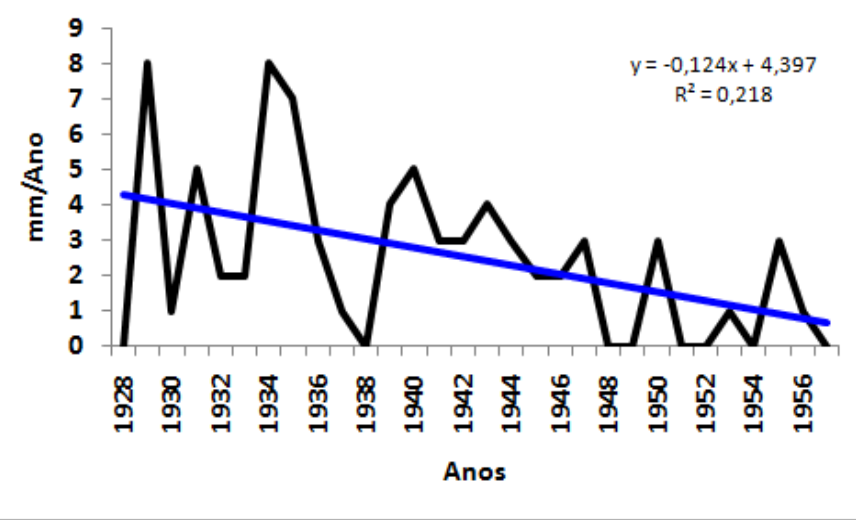

(b) R20mm: Cabaceiras (PB) - Período 1958/1987

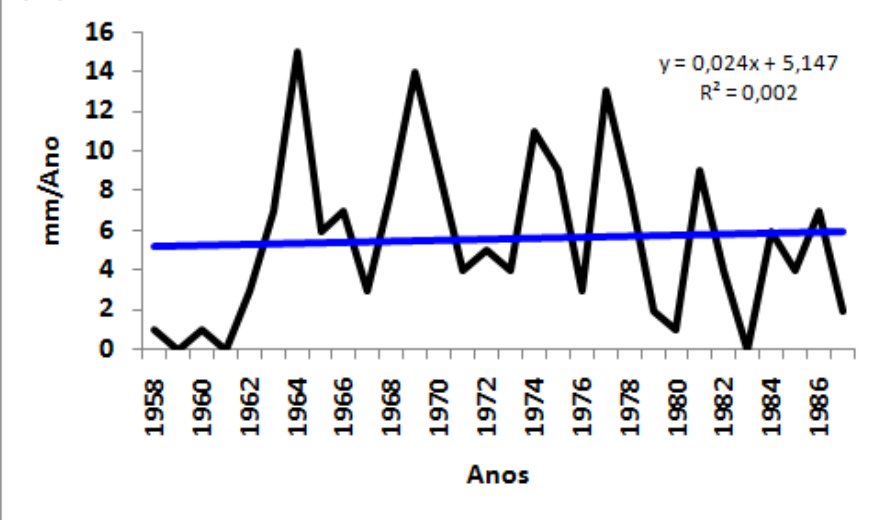

(c) R20mm: Cabaceiras (PB) - Período 1988/2017

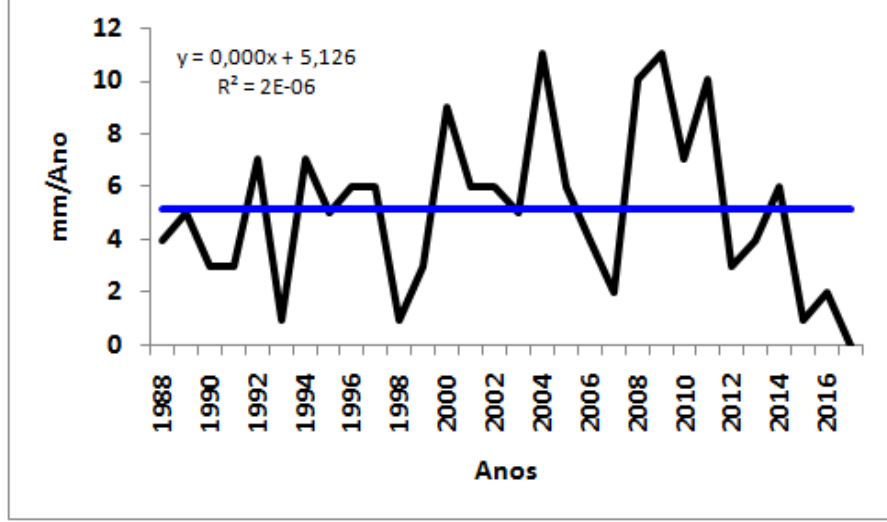

(d) R20mm: Cabaceiras (PB) - Período 1928/2017

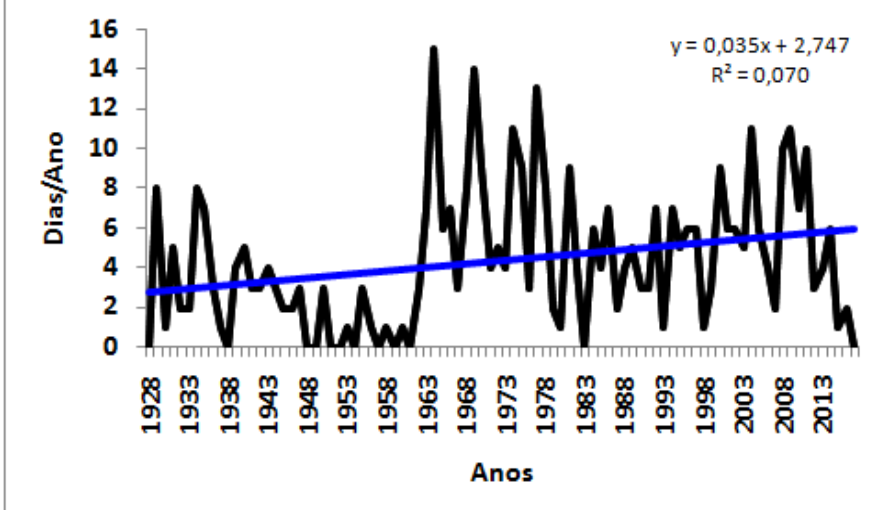

Figura 4. Índice R20mm para os subperíodos (a)1928-1957, (b)1958-1987 e (c)1988-2017, e para o período total (d)1928-2017. 

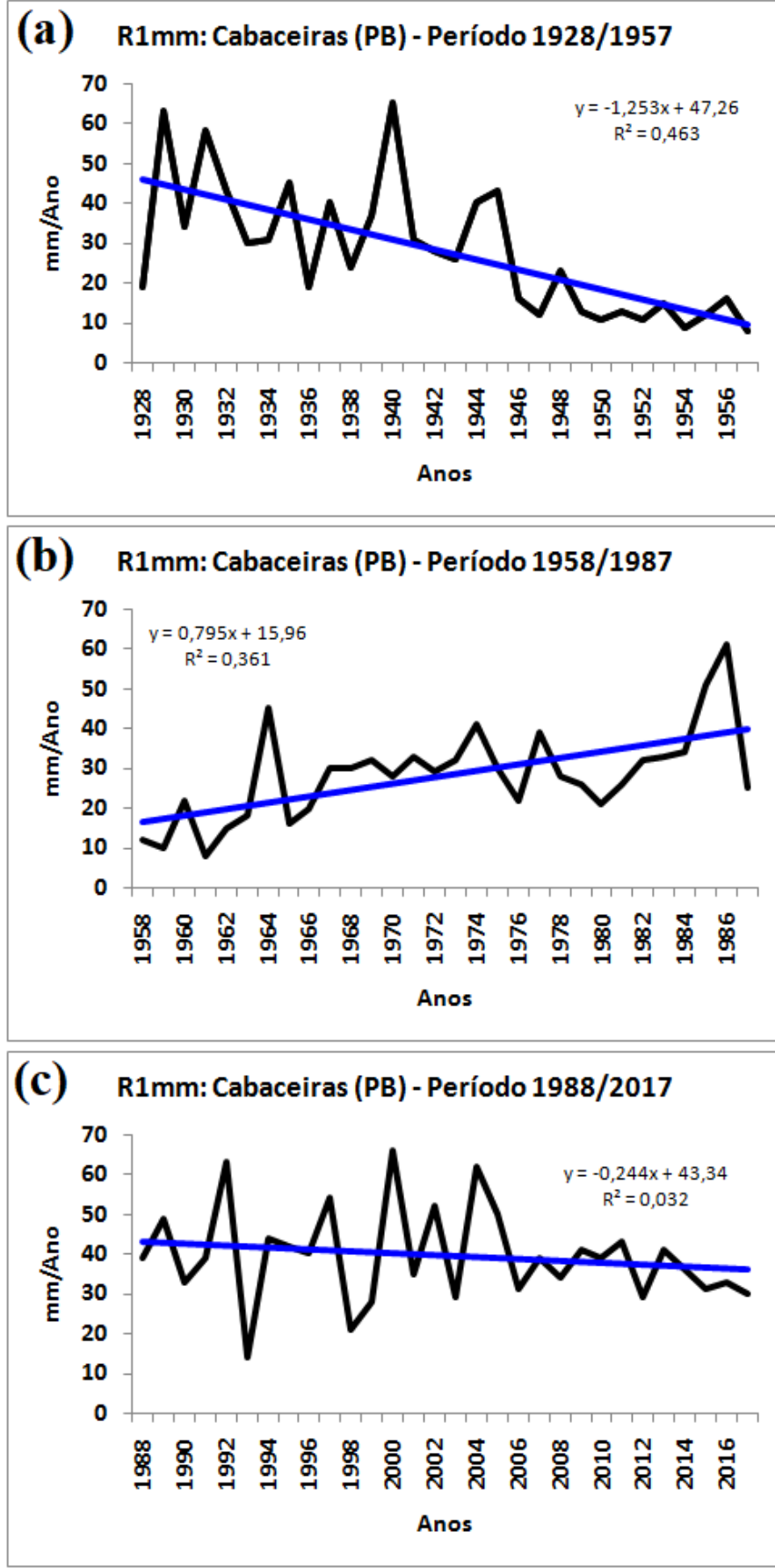

(d) R1mm: Cabaceiras (PB) - Período 1928/2017

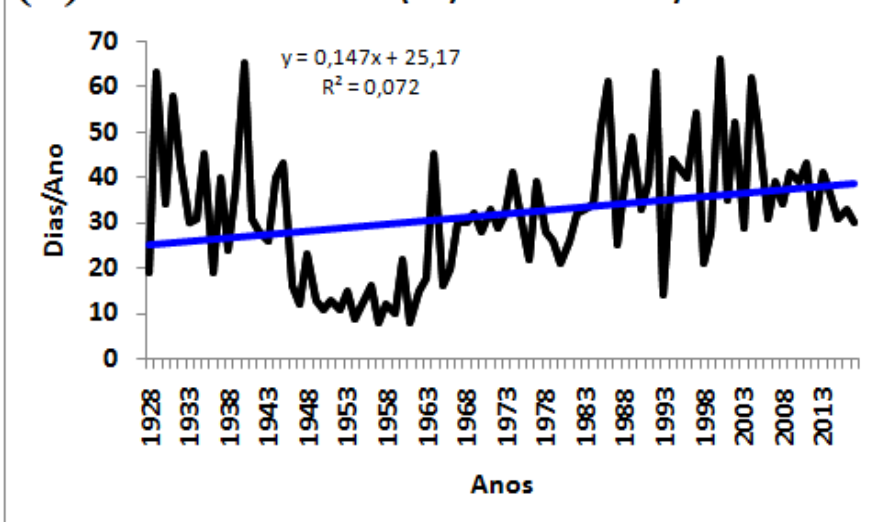

Figura 5. Índice R1mm para os subperíodos (a)1928-1957, (b)1958-1987 e (c)1988-2017, e para o período total (d)1928-2017. 

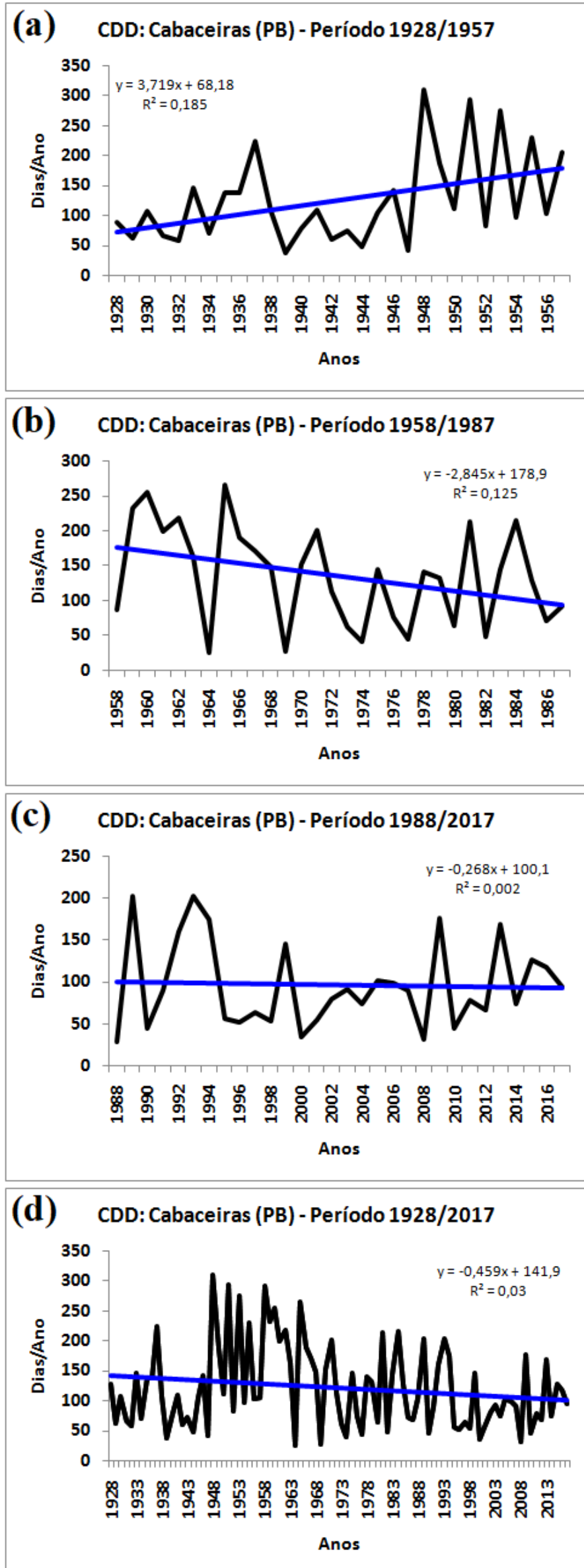

Figura 6. Índice CDD para os subperíodos (a)1928-1957, (b)1958-1987 e (c)1988-2017, e para o período total (d)1928-2017. 

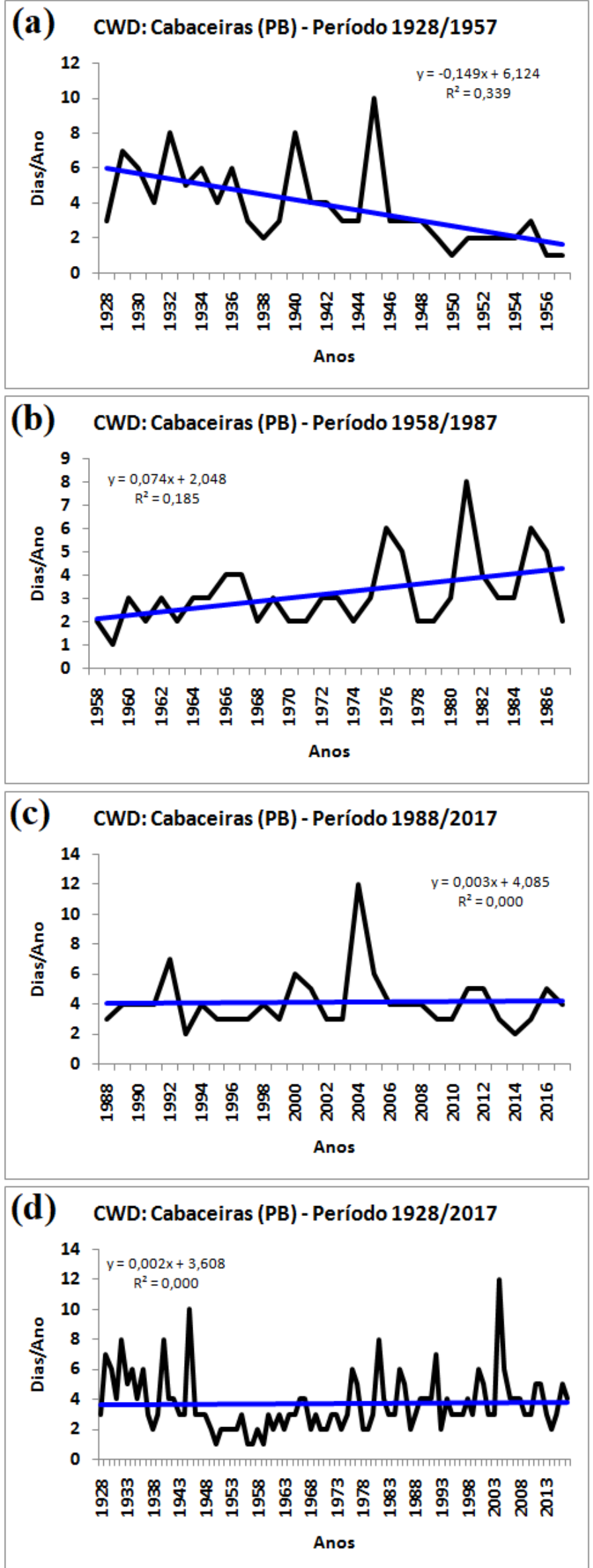

Figura 7. Índice CWD para os subperíodos (a)1928-1957, (b)1958-1987 e (c)1988-2017, e para o período total (d)1928-2017. 


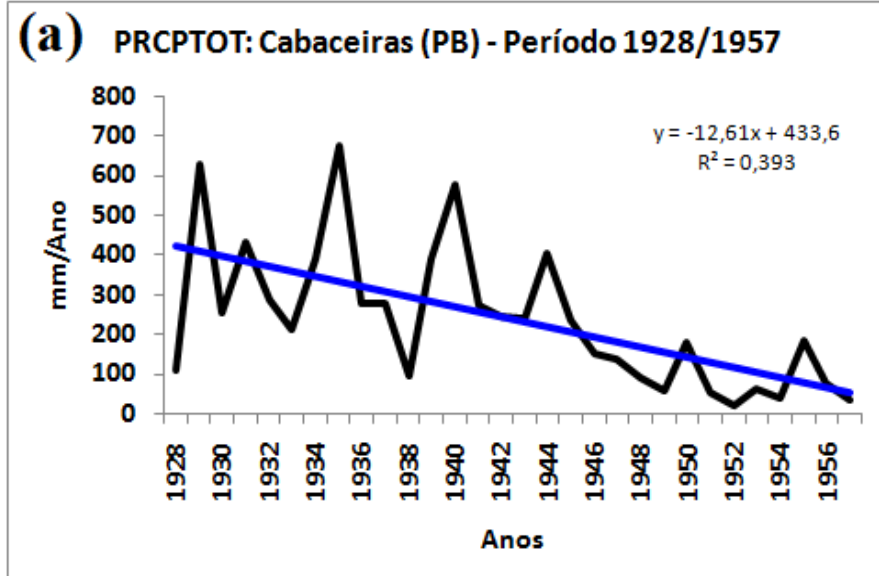

(b) PRCPTOT: Cabaceiras (PB) - Período 1958/1987

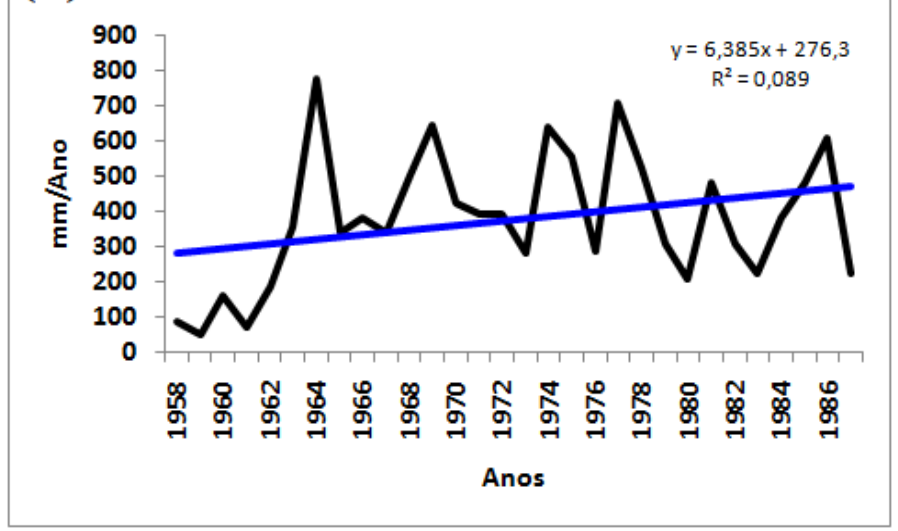

(c) PRCPTOT: Cabaceiras (PB) - Período 1988/2017

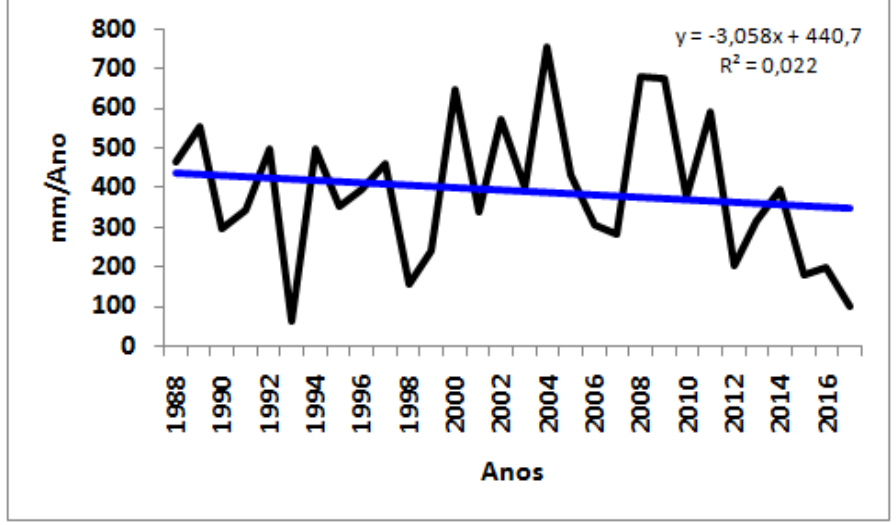

(d) PRCPTOT: Cabaceiras (PB) - Período 1928/2017

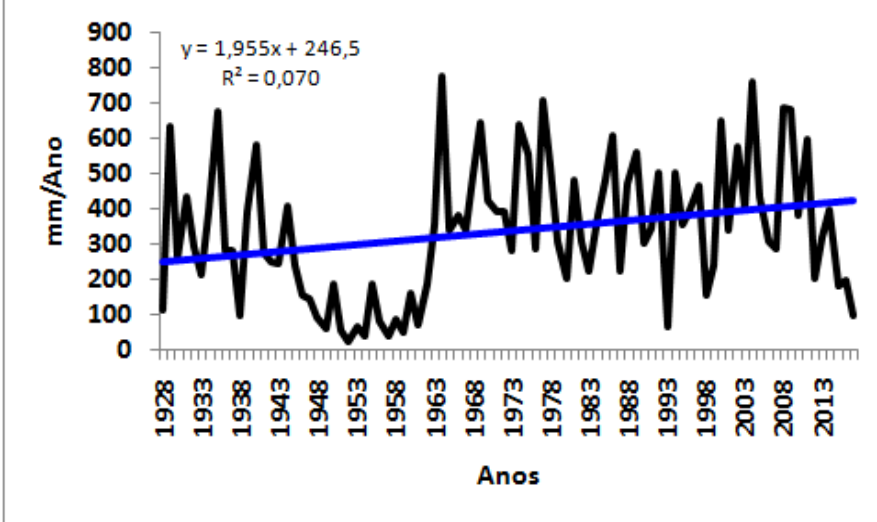

Figura 8. Índice PRCPTOT para os subperíodos (a)1928-1957, (b)1958-1987 e (c)1988-2017, e para o período total (d)1928-2017. 


\section{Conclusões}

Os resultados desta breve pesquisa sobre tendências de índices de extremos de precipitação indicam que deve-se ter muita cautela ao afirmar categoricamente que um determinado índice apresenta tendência positiva/negativa com significância estatística.

Deve ser ressaltado que esta tendência reflete uma situação correspondente apenas ao período de dados estudado, ou seja, indica uma situação particular de um momento do tempo cronológico que pode não refletir o todo.

Este tipo de abordagem é mais fácil de ser compreendida ao se analisar séries temporais longas que possam ser divididas em subperíodos de, ao menos, 30 anos, como foi o caso desta pesquisa para cabaceiras-PB.

Alguns dos índices analisados, citando o CDD e CWD como exemplo, mostram claramente tendências opostas entre os períodos 1928-1957 e 1958-1987, estatisticamente significativas, e pouca ou irrelevante tendência no segmento final da série entre 1988-2017. O mesmo ocorre com outros índices não citados, incluindo-se todos os que não foram descritos nos resultados por não apresentarem significância estatística nas tendências de qualquer período.

De um modo geral, analisando-se o comprimento total da série de dados, as tendências dos índices foi positiva, com exceção do CDD, o que demonstra que a região de Cabaceiras atravessou um período úmido mais constante a partir da década de 1960 até 2010, após o longo período de estiagem entre as décadas de 1940 e 1950, com o período recente de secas de 2012-2017 influenciando pouco nos valores dos índices de extremos para o período total da série.

\section{Agradecimentos}

$\mathrm{O}$ primeiro autor agradece à Coordenação de Aperfeiçoamento de Pessoal de Nível Superior pela concessão da Bolsa de Pós-Doutorado Júnior, o quarto autor agradece à Coordenação de Aperfeiçoamento de Pessoal de Nível Superior pela concessão da Bolsa de Mestrado, e os quintos e sexo autores agradecem as bolsas de iniciação científica concedidas pela UFAL/CNPq/FAPEAL.

\section{Referências}

Alves, J.M.B., Silva, E.M., Sombra, S.S., Barbosa, A.C.B., Santos, A.C.S., Lira, M.A.T., 2017. Eventos Extremos Diários de Chuva no Nordeste do Brasil e Características Atmosféricas. Revista Brasileira de Meteorologia 32, 227-233.
Alves, T.L.B.; Azevedo, P.V.; Santos, C.A.C., 2017. Influence of climate variability on land degradation (desertification) in the watershed of the upper Paraíba River. Theoretical and Applied Climatology 127, 741-751.

Alvala, R.C.S., Cunha, A.P.M.A., Brito, S.S.B., Seluchi, M.E., Marengo, J.A., Moraes, O.L.L., Carvalho, M.A., 2017. Drought monitoring in the Brazilian Semiarid region. Anais da Academia Brasileira de Ciências, 91, 1-15.

Baez-Gonzaleza, A.D., Torres-Mezab, M.J., RoyoMarquezc, M.H., Kiniryd, J.R., 2018. Climate variability and trends in climate extremes in the priority conservation area El Tokio and adjacent areas in northeastern Mexico. Weather and Climate Extremes 22, p. 36-47.

Carvalho, J.R.P., Nakai, A.M., Monteiro, J.E.B.A., 2016. Spatio-Temporal Modeling of Data Imputation for Daily Rainfall Series in Homogeneous Zones. Revista Brasileira de Meteorologia 31, 196-201.

Croitoru, A-E., Piticar, A., Burada, D. C., 2016. Changes in precipitation extremes in Romania. Quaternary International 415, 325-335.

Cunha, A.P.M.A., Alvala, R.C.S., Nobre, C.A., Carvalho, M.A., 2015. Monitoring vegetative drought dynamics in the Brazilian Semiarid Region. Agricultural and Forest Meteorology 214-215, 494-505.

Cunha, A.P.M.A., Tomasella, J., Ribeiro-Neto, G.G., Brown, M., Garcia, S.R., Brito, S.B., Carvalho, M.A., 2018. Changes in the spatialtemporal patterns of droughts in the Brazilian Northeast. Atmospheric Science Letters 19, 18.

Dantas, L.G., Santos, C.A.C., Olinda, R.A., 2015. Tendências anuais e sazonais nos extremos de temperatura do ar e precipitação em Campina Grande - PB. Revista Brasileira de Meteorologia 30, 423 - 434.

Geirinhas, J.L., Trigo, R.M., Libonati, R., Coelho, C.A.S., 2017. Climatic and synoptic characterization of heat waves in Brazil. International Journal of Climatology 1, 1-17.

Guimarães, S.O., Costa, A.A., Vasconcelos Júnior, F.C., Silva, E.M., Sales, D.C., Araújo Júnior, L.M., Souza, S.G., 2016. Projeções de Mudanças Climáticas sobre o Nordeste Brasileiro dos Modelos do CMIP5 e do CORDEX. Revista Brasileira de Meteorologia 31, 337-365.

IPCC. 2013. Climate Change 2013 - The Physical Science Basis. Working Group I Contribution to the IPCC Fifth Assessment Report. Summary for Policymakers. IPCC, Stockholm, Sweden.

Jiang, D., Wang, K., Li, Z., Wang, Q., 2011. Variability of extreme summer precipitation 
over Circum-Bohai-Sea region during 19612008. Theoretical and Applied Climatology 104, 501-509.

Keggenhoff, I., Elizbarashvili, M., AmiriFarahani, A., King, L., 2014. Trends in daily temperature and precipitation extremes over Georgia, 1971-2010. Weather and Climate Extremes 4, 75-85.

Marengo, J.A., Alves, L.M., Alvalá, R. C.S., Cunha, A.P.M.A., Brito, S.S.B., Moraes, O.L.L., 2018. Climatic characteristics of the 2010-2016 drought in the semiarid Northeast Brazil region. Anais da Academia Brasileira de Ciências 90, 1973-1985.

Martins, E.S.P.R., Coelho, C.A.S., Haarsma, R., Otto Fel, King, A.D., Oldenborgh, G.J.V., Kew, S., Philip, S., Vasconcelos Junior, F.C., Cullen, H., 2018. A Multimethod Attribution Analysis of the Prolonged Northeast Brazil Hydrometeorological Drought (2012-16). Bulletin of the American Meteorological Society 99, 65-69.

Martins, M.A., Tomasella, J., Rodriguez, D.A., Alvala, R.C.S., Giarolla, A., Garofolo, L.L., Siqueira Júnior, J.L., Paolicchic, L.T.L.C., Pinto, G.L.N., 2018. Improving drought management in the Brazilian semiarid through crop forecasting. Agricultural Systems 160, 21 30.

Medeiros, R.M., Brito, J.I.B., Borges, C.K., 2012. Análise Hidroclimático do Município de Cabaceiras, PB. Revista Brasileira de Geografia Física 05, 1174-1190.

Natividade, U.A., Garcia, S.R., Torres, R.R., 2017. Tendência dos Índices de Extremos Climáticos Observados e Projetados no Estado de Minas Gerais. Revista Brasileira de Meteorologia 32, 600-614.

Nóbrega, J.N., Santos, C.A.C., Gomes, O.M., Bezerra, B.G., Brito, J.I.B., 2014. Eventos Extremos de Precipitação nas Mesorregiões da Paraíba e suas Relações com a TSM dos Oceanos Tropicais. Revista Brasileira de Meteorologia 29, 197 - 208.

Nóbrega, R.S., Farias, R.F.L., Santos, C.A.C., 2015. Variabilidade temporal e espacial da precipitação pluviométrica em Pernambuco através de índices de extremos climáticos. Revista Brasileira de Meteorologia 30, 171-180.
Santos, C.A.C., Brito, J.I.B., 2007. Análise dos Índices de Extremos para o semi-árido do Brasil e suas Relações com TSM e IVDN. Revista Brasileira de Meteorologia 22, 303-312.

Santos, C.A.C., Brito, J.I.B., Rao, T.V. R., Menezes, H.E.A., 2009. Tendências dos índices de precipitação no Estado do Ceará. Revista Brasileira de Meteorologia 24, 39-47.

Santos, C.A.C., Oliveira, V.G., 2017. Trends in Extreme Climate Indices for Pará State, Brazil. Revista Brasileira de Meteorologia 32, 13-24.

Silva, A.G., Azevedo, P., 2008. Índices de tendências de Mudanças Climáticas no Estado da Bahia. Engenharia Ambiental 5, 141-151.

Silva, M.T., Silva, V.P.R., Azevedo, P.V., 2012. O cultivo do algodão herbáceo no sistema de sequeiro no Nordeste do Brasil, no cenário de mudanças climática. Revista Brasileira de Engenharia Agrícola e Ambiental 16, 80-91.

Tank, A.M.K., Zwiers, F.W., Zhang, X., 2011. Guidelines on analysis of extremes in a changing climate in support of informed decisions for adaptation. WMO.

Thornthwaite, C.W.; Mather, J.R., 1955. The water balance. Publication in Climatology, 8, Laboratory of Climatology, Centerton, N.J.

Wanderley, H.S., Justino, F.B., Sediyama, G.C., 2016. Tendência da Temperatura e Precipitação na Península Antártica. Revista Brasileira de Meteorologia 31, 114-121.

Zhang, X., Yang, F., 2004. RClimDex (1.0) User Manual. Climate Research Branch Environment Canada, Ontario, Canada.

Zhang, X., Alexander, L.V., Hegerl, G.C., KleinTank, A., Peterson, T.C., Trewin, B., Zwiers, F.W., 2011. Indices for monitoring changes in extremes based on daily temperature and precipitation data. Wiley Interdisciplinary Reviews: Climate Change 2, 851-870.

Zwiers, F.W., Alexander, L.V., Hegerl, G.C., Knutson, T.R., Naveau, P., Nicholls, N., Schär, C., Seneviratne, S.I., Zhang, X., 2011. Community paper on climate extremes. Challenges in Estimating and Understanding Recent Changes in the Frequency and Intensity of Extreme Climate and Weather Events, World Climate Research Programme Open Science Conference, 24-28 October 2011, Denver, CO, USA. 